\title{
Novel Method to Teaching Electrical and Computer Engineering Courses by a Modern Multimedia-Based Approach
}

\author{
Raymond Phan \\ Ph.D. Student - Department of Electrical and Computer Engineering \\ Ryerson University \\ rphan@ee.ryerson.ca*
}

\begin{abstract}
This paper presents a novel approach to Electrical and Computer Engineering education at Ryerson University. First, the board is eliminated all together, using a Tablet PC. Handwriting recognition software is used to display any notes and diagrams that were originally intended on a board. Next, the lecture has its desktop video, and audio through a microphone, captured in real-time using CamStudio, an open source video capturing tool, while simultaneously addressing the class. After, the video captures are placed on a public video sharing website, where students may access the videos at any time, to review lectures as if they were physically in the lecture on that day. The overall averages for the courses currently taught by the author, in comparison to the previous years, has raised by roughly $8 \%$, demonstrating that this method of education is sufficiently better, and significantly improves the learning process.
\end{abstract}

\section{Introduction}

Engineering education in the early $20^{\text {th }}$ century was delivered using a textbook and notetaking approach. With the introduction of the

\footnotetext{
*Address: 350 Victoria St., Toronto ON, M5B 2K3, Canada
}

Internet, engineering education has revolutionized, primarily due to its strong support for breaking the boundaries of the traditional approach. Electronic copies of course materials are placed on web pages specifically designed by the instructor of the course. However, this can become tedious, as the web pages can become difficult to maintain. Therefore, course management systems (CMS) were created, which alleviate the amount of work involved to maintain a course webpage, and providing support for students. In order to design a course webpage, CMSs provide a simple user interface to construct these webpages. Without any knowledge of programming languages, instructors can create sophisticated webpages with only a few mouse clicks. Most universities and colleges now offer course materials over course management systems, such as BlackBoard [1].

Though this provides good support, it has some limitations, especially if the student misses a class. If lectures were missed, there would be no way to view the lecture after it has taken place, and students could only rely on notes made by students, or if the instructor posts notes. Even in this situation, there are statements and references that the instructor made in class that were not captured, and there is no guarantee that a student will write 
these references. Therefore, in addition with CMSs, lectures are video captured in real-time and are posted immediately after. In this case, everything in the lecture is captured. Currently, there are solutions that exist that provide realtime video capturing of lectures, such echo ${ }^{360}$ [2], and are used throughout the world. However, these solutions are not free, and can only be used at institutions who have purchased the corresponding licenses. Therefore, this paper presents a method to teach engineering courses using this same framework, but by only using free and open-source tools. The paper illustrates a free replication of the same solution as seen in echo ${ }^{360}$, to video capture lectures in real-time, and to provide them online on the Internet. It should be noted that this is a solution for desktop capturing, which performs real-time video capturing of a computer desktop. This method does not perform video capturing of the actual instructor in front of a board, as this is obviously not free. Desktop capturing is the best way to perform real-time video capturing, without using any proprietary equipment or software. In addition, desktop capturing was the method chosen, as diagrams, graphs and equations are heavily used in engineering education, and the desktop is projected on a projector. The desktop and projector are available in most college and university lecture rooms. The figures can quickly be imported and shown on a desktop, rather than drawing them on a board. In addition, the method can also be performed outside of the lecture room, as all of the components are open-source. If the instructor were to post additional tutorial questions, or corrections, outside of the scheduled lecture time, they can do so in the comfort of their own home, or office, and can post the videos accordingly, independent of being present in the classroom.

The rest of this paper is as follows. Section 2 outlines the overall process to perform realtime desktop video capturing, starting from the components used in performing this task, to generating a video file at the end of a lecture. Finally, Section 3 illustrates some results using this method, including some sample snapshots, and a comparison of student averages of the courses taught by the author using the proposed method, in comparison to previous years. Finally, Section 4 will conclude the paper, and illustrate future work in this area.

\section{Overall Process}

Essentially, a computer and a projector is used to project content in a classroom. The video capture process is simultaneously run while lecturing. As the class is addressed, the audio from the lecture is captured with the same solution while speaking into a microphone. After the lecture, the video capture is saved and a .AVI file is produced. This file is published on the Internet for public access and usage. Each following subsection will explain this process in further detail.

\subsection{Hardware}

For this approach, in order to perform desktop capturing, a PC or laptop with moderate specifications is required. At Ryerson University, most of the lecture rooms are equipped with the current state-of-the-art PCs, connected to a digital projector with a good resolution. However, the author has chosen to use a Tablet $P C$, which is a combination of a laptop and a WACOM tablet. The WACOM tablet is integrated into the laptop, and is overlayed on top of the screen. Tablet PCs are accompanied with a specialized stylus pen, and with some sort of handwriting recognition software. This allows the user to make handwritten notes on the laptop, by writing directly onto the screen. As such, instead of using the desktop that is available in the lecture room, the tablet PC is projected instead. The main reason why the tablet PC is used is because written lecture notes can be shown on the projector, which 
honours the traditional style of teaching engineering courses with a board, but at the same time, graphs, diagrams, and figures can be easily imported and shown on the screen. Past figures can be referred to by simply scrolling up on the page, rather than redrawing the figure on the board. In addition, Java applications and Internet webpages can be shown using the same platform, without having to refer back and forth between the board, and the computer. Essentially, every task can be performed on the tablet PC itself. A state-of-the-art tablet PC is not required for this approach; the author uses a Toshiba Portege M300, equipped with 1 GB of RAM, a Pentium 4 Mobile $1.7 \mathrm{GHz}$ processor, and with the Windows XP Tablet Edition operating system. The tablet PC is well over 5 years old, but still performs well for this process. The handwriting recognition software that is used is Windows Journal, which is accompanied with the tablet edition of Windows XP. It should be noted that a desktop can still use the same approach, and either a stylus pad can be attached to the desktop, or PowerPoint presentations can be captured.

\subsection{Desktop Capturing Software}

As mentioned earlier, most real-time video capturing solutions require a license to be purchased to use the software. In order to promote free solutions, the author uses an open-source real-time video capturing tool called CamStudio [3], which is a free real-time desktop video capturing solution developed in Visual $\mathrm{C}++$. As this is developed in Visual $\mathrm{C}++$, this solution is not compatible with Linux or Mac OS X operating systems. CamStudio has the ability to record all desktop screen activity on a PC, to create industry-standard .AVI video files. In addition, the tool can capture input from an audio device, such as a microphone. Therefore, during the lecture, the video capturing software is run. The movements on the tablet PC screen are also projected in the classroom, as well as being captured by the software. As the classroom is being addressed, the audio is captured by a simple analog microphone headset, which is plugged into the microphone input of the tablet PC. The capturing software will capture the audio, and embed it with the video capture. Essentially, the output video will be a frame-by-frame reconstruction of the lecture seen in the point of view of the projector, where each stroke made on the tablet PC is in synchronization with the audio that accompanied the corresponding strokes. The audio capture settings established with CamStudio are the following: 1) Record audio from microphone, 2) Audio Options for Microphone: a) Audio Capture Device: The default device (whatever it may be), b) Recording Format: 11.025 kHz, mono, 8-bit, c) PCM as the Compressed Format, d) Interleave video and audio every $100 \mathrm{~ms}$.

\subsection{Video Compression Codec}

Most lectures are one hour long, or if they are more than one hour long, there are breaks in between. Typically, for the classes that the author teaches, if the lectures are longer than one hour, the lecture videos are split up into one hour segments. When performing a desktop capture, it should produce a video file of a decent size. Using no video compression is unacceptable, as uncompressed video that is an hour long would result in large and unacceptable file sizes for public access. Therefore, some sort of compression scheme, or codec, should be used in order to maintain roughly the same amount of quality, but encouraging a smaller file size. A codec that falls into the free and open-source category should be used. The codec that was ultimately used in this framework was the $X v i D$ codec [4]. Therefore, each frame that is captured from the desktop is compressed using the XviD compression scheme, and the final video file is generated by the XviD codec. There are similari- 
ties between the XviD codec, and the popular DivX codec [5]. They are both based off of the MPEG-4 standard. However, DivX is not open-source software, which means that support for the codec is only provided for certain operating systems. In addition, only the decoder is provided for free, which is used for playing DivX video files. However, the encoder must be purchased, which is responsible for transforming raw video into the DivX format. XviD is open-source, and both the encoder and decoder are free. Therefore, XviD is the method of choice on a free and opensource viewpoint. In order to ensure a decent file size for the resultant video, the resolution chosen was $1024 \times 768$, as a resolution that is too large will render the file size to be larger, and a resolution to be too small would make lecturing uncomfortable, as there would not be enough room to illustrate figures and write out lecture notes. The video capture settings set with CamStudio are the following: 1) Quality: 100, 2) Auto adjust frame rate, 3) Codec configurations: a) Unrestricted profile, b) Single pass encoding type, c) Target quantizer: 4.00 , d) First frame weighting at 1.00, e) Quality preset: General purpose. Using these settings, the average file size for a one hour lecture ranges between 140 to $180 \mathrm{MB}$. This file size that is most suitable for public access, and can be downloaded in a matter of minutes using the average Internet connection.

\subsection{Publishing Mediums}

Once the video file(s) have been generated, they should be made available for public access. Public video sharing websites appeal the most for this process, due to the fact that videos can be posted at anytime, and is independent of the instructor being present in the classroom. In addition, membership to these websites are free, which is appealing in this free and opensource framework. As such, not only can this be used to capture desktop video during the lecture, but can capture desktop video outside of the lecture, if additional tutorial questions, or corrections to the lectures are desired to be published. To date, YouTube [6] is the most popular video sharing website on the Internet. Visitors who wish to view a video on YouTube go to a dedicated link used to host the particular video. Videos are uploaded to the website, and the video is transcoded and converted into the Adobe Flash Player format, which is crossplatform and freely available [7]. The only requirement to view this format is that a plugin be installed, and the plugin exists for any operating system that exists. Therefore, each lecture, or each part of a lecture, would have its own dedicated web link, independent of the other lectures. However, YouTube does not allow long videos to be uploaded, and only allow a maximum of 10 minutes per video. Though taking a one hour lecture, and splitting them up into 10 minute portions is possible, it will be distracting towards the overall lecture experience, as the video should mimic the experience in class, without any frequent 10 minute "breaks". Therefore, another public video sharing website was sought out, and blip.tv [8] was the service that was ultimately used in this approach. blip.tv also uses the Adobe Flash Player format, but only has a file size limitation, which is $1 \mathrm{~GB}$. The video file sizes generated within this framework are significantly below this requirement, and since there is no length limitation, this website proves to be attractive for hosting lecture videos. In addition, the actual .AVI files of the lectures can be downloaded off of blip.tv, which further increases its attractiveness for public video sharing. The website for the author's lectures can be found at: http: / / rayphan.blip.tv.

In addition, Ryerson University uses the BlackBoard CMS to provide online support and management of courses. The author uses the BlackBoard CMS to post the course outline, solutions to assignments, midterms and 
quizzes, laboratory session documents, and other related material. The handwritten notes that were made on the tablet PC are also provided on BlackBoard in PDF format, as well as the actual video files themselves. As such, students enrolled in the course are not required to write any notes in class, and can access these notes after each lecture. However, these notes are not posted publicly, in order to prevent any modifications, and for reposting the notes without any credit. Since the video content cannot be modified, there is no fear for posting the videos publicly, as the videos are protected by the Creative Commons Attributions 3.0 License [9].

\section{Results}

\subsection{Student Averages}

During the academic year of 2009, the author taught two upper-year undergraduate electrical engineering courses at Ryerson University: ELE 635 - Communication Systems, and ELE 639 - Control Systems. Table 1 represents the overall averages for both courses over a five year period, between 2005 and 2009. Between 2005 and 2008, different instructors were teaching the courses, and in the 2009 academic year, the author was the only instructor for both courses, and was his first year as a sessional instructor. Only the Winter term averages are shown, as they are a true reflection of the performance of the students. The summer term offers the same courses, but are for students who are taking the courses for remedial reasons. The courses during these years have not changed, and the same difficulty of the material and style of the evaluations was maintained.

As seen in Table 1, using the proposed approach has significantly improved the overall average. This is most likely due to the fact that students are able to refer to any lecture on their own time, and can refer to any part of the lec-

\begin{tabular}{|c|c|c|}
\hline Course & Year & Overall Average \\
\hline ELE 635 & 2005 & $68.6 \%$ \\
\hline ELE 635 & 2006 & $67.5 \%$ \\
\hline ELE 635 & 2007 & $69.4 \%$ \\
\hline ELE 635 & 2008 & $70.1 \%$ \\
\hline ELE 635 & $\mathbf{2 0 0 9}$ & $\mathbf{7 7 . 4 \%}$ \\
\hline ELE 639 & 2005 & $70.9 \%$ \\
\hline ELE 639 & 2006 & $72.4 \%$ \\
\hline ELE 639 & 2007 & $71.7 \%$ \\
\hline ELE 639 & 2008 & $71.4 \%$ \\
\hline ELE 639 & $\mathbf{2 0 0 9}$ & $\mathbf{7 9 . 6 \%}$ \\
\hline
\end{tabular}

Table 1: Table of overall averages for ELE 635 and ELE 639 over a 5-year period.

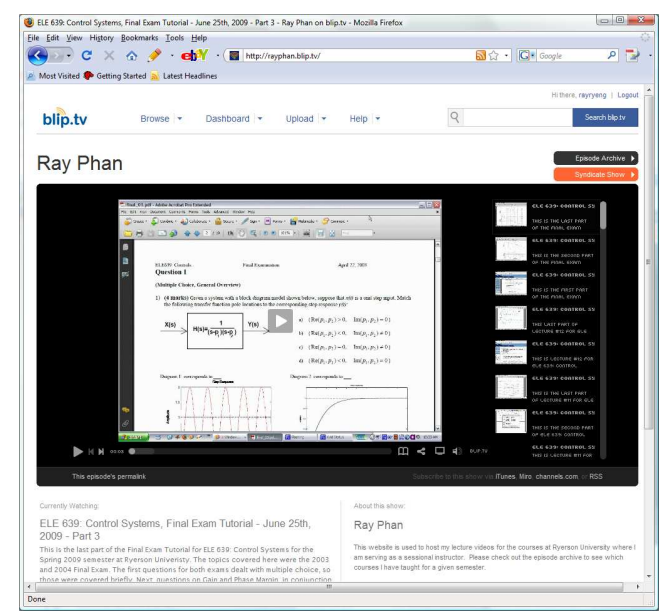

Figure 1: Welcome page of http: / / rayphan.blip.tv

ture whenever they desire. If students were to ever miss the lecture, they would be able to see the lecture on their own time, and be able to view the lecture in the same fashion as those who were actually present.

\subsection{Sample Snapshots}

Figs. 1 and 2 illustrates some snapshots of the public video sharing website that is used to share the video lectures made by the author.

Fig. 1 illustrates the welcoming page of the website, showing the latest uploaded lecture in the Adobe Flash Player format. The 


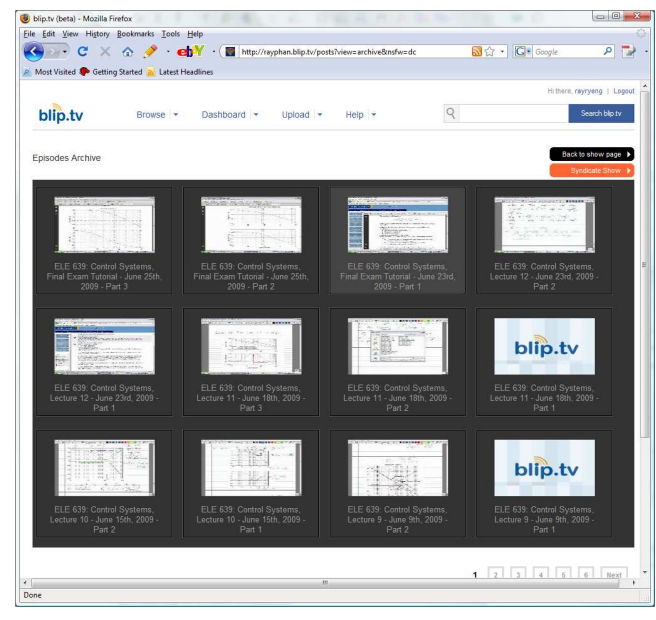

Figure 2: Episode Archive page of http://rayphan.blip.tv

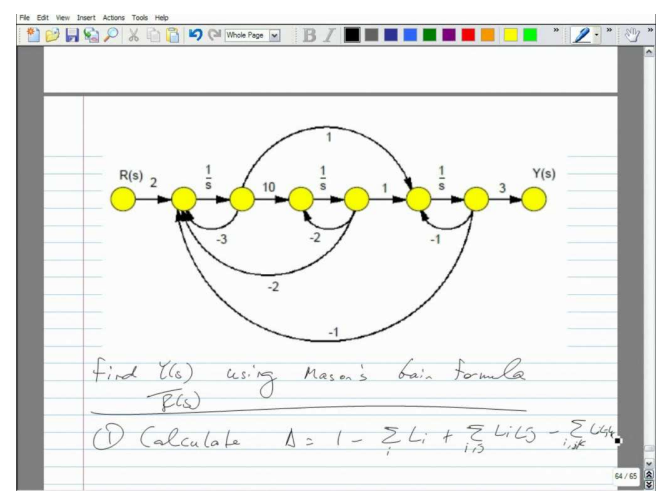

Figure 3: Sample shot of a ELE 639 lecture

user has the option of viewing the lecture full screen, or a windowed version. Fig. 2 illustrates the "Episode Archive", which shows a list of all of the lectures in descending order of upload. This can be accessed through the Episode Archive link on the webpage. Students can view any lecture in the course by clicking on the corresponding thumbnail. Fig. 3 represents a full screen shot of a lecture from ELE 639. The quality of the captures is quite good, even when a high compression is observed from the resulting video file.

\section{Conclusions}

This paper presented a method for capturing desktop content in real-time using only free and open-source methods. Lectures are presented in the classroom using a tablet PC, or desktop, and is projected on a projector in a classroom. The desktop of the tablet PC is video captured using CamStudio, an opensource desktop recording tool, and simultaneously records the audio while addressing the class. The end product is a video file that is in synchronization with the strokes made on the tablet PC, with the audio that accompanies each stroke. These video files are posted on blip.tv, a public video sharing website, where students are able to access the videos outside of the lecture, to review any lectures as if they were physically there on that day. The overall averages for the courses that were taught by the author, in comparison to the previous years, has raised roughly $8 \%$, demonstrating that this method of education is sufficiently better, and significantly improves the learning process.

\section{References}

[1] BlackBoard Course Management System http://www.blackboard.com.

[2] $\mathrm{Echo}^{360}$ Inc. The EchoSystem http:// www.echo360.com.

[3] CamStudio ${ }^{T M}$ : OpenSource Free Streaming Video Software http:// www.camstudio.org.

[4] XviD Codec http://www.xvid.org.

[5] DivX Codec http://www.divx.com.

[6] YouTube http://www.youtube.com.

[7] Adobe Flash Player http://www.adobe.com.

[8] Blip Networks Inc. http://www.blip.tv.

[9] Creative Commons http://www.creativecommons.org. 